\title{
The Swept Surface of an Elliptic Cylinder \\ SM-01-020
}

\author{
Stephen Mann \\ smann@@cgl.uwaterloo.ca \\ Sanjeev Bedi \\ Computer Science Department \\ sbedi@uwaterloo.ca \\ University of Waterloo \\ Waterloo, Ontario, N2L 3G1 CANADA
}

\begin{abstract}
In this paper, we present a method for computing a piecewise linear approximation to the surface swept by a moving, rotating elliptic cylinder. Our method is a generalization of the imprint point method we developed for computing points on a surface of revolution. The method is based on on identifying grazing points on the surface of revolution at a sequence of positions, and for each position connecting the grazing points with a piecewise linear curve. A collection of grazing curves is joined to approximate the swept surface and stitched into a solid model. Previously this method has been tested on cylinders, toruses, and cones.

This work extends the imprint point method to compute the swept surface generated by a general closed surface. The method is demonstrated by translating and rotating an elliptic cylinder along a spline curve. The application of this method is in interference detection of moving machinery such as robots. It is anticipated that a robot would be modeled as a collection of components enclosed in elliptic cylinders, cones, toruses and spheres. Our method can be used to generate the swept volume of these enclosing objects, and used for interference detection and avoidance.
\end{abstract}

\section{Introduction}

Swept surface are frequently needed in many engineering applications. Swept volumes are important for determining interference free trajectories for robots and for generation of gouge free tool paths. The existing methods rely on SEDE [1] or envelop theory [2,3]. Implementation of these techniques results in simultaneous solution non-linear equations which can be tedious to generalize and time consuming to compute.

A new method was presented for the creation of swept surfaces swept by surfaces of revolution. This method is similar to envelop theory, but uses some surface properties to speed up the calculation. The idea is based on the observation that when a solid cylinder translates it sweeps a surfaces made up of straight lines. To find points on the straight lines, the cylinder can be imagined to be made of a stack of disks and each disk translates in the same direction. Then the grazing point on one disk on the surface at a particular tool position can be identified by taking a cross product of the normal to the disk with the direction of motion. This basic idea can be extended to a cylinder executing simultaneous translation and rotation, although in this case the grazing curves are not straight lines but curves on the cylinder. The cross product method works for tori and spheres; a more general method can be applied to find grazing curves on an arbitrary surface of revolution, which we review in the next section.

In this paper, we further extend the method to determine the surfaces swept by straight and twisted elliptic cylinders (Figures 4 and 5). The concept can be generalized to convex closed surfaces, but examples in this paper will be limited to the elliptic case.

In the next section, we will review grazing curves and our earlier work on computing the surface swept by an APT cutter (i.e., a surface of revolution). Then in Section 3, we extend this work to elliptic cylinders, giving examples in Section 4 .

Submitted to the Sixth ACM Symposium on Solid Modeling and Applications, Ann Arbor, Michigan, June 4-8, 2001 

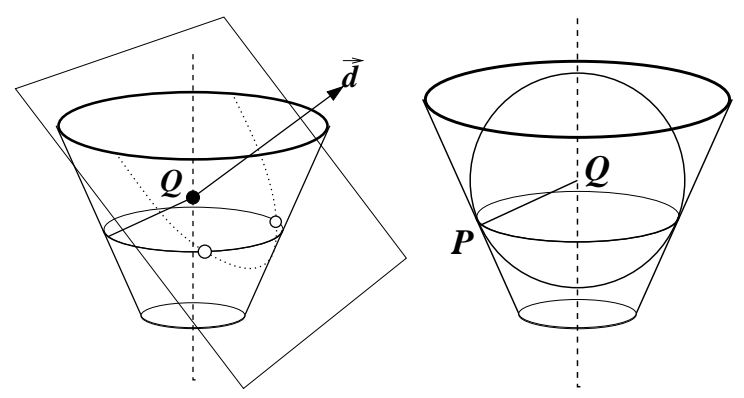

Figure 1: Computing grazing points.

\section{Grazing Curves and Swept Surfaces}

Chung, Park, Shin and Choi [4] present a method for determining the surface swept by a generalized APT cutter for 3-axis machining. The generalization of Chung et al.'s method to 5-axis machining is non-trivial. In an earlier paper [5], we defined an grazing curve in 5-axis machining as the set of points on the rotating tool surface at which the direction of motion lies in the tangent plane of the cutter. The silhouette curve used in 3-axis machining is a special case of a grazing curve where there is no rotation.

The method for any surface of revolution is as follows. The tool (which is a surface of revolution) is sliced with planes perpendicular to the tool axis. Each plane slices the tool in a circle. On each circle, we compute 0, 1, or 2 grazing points. The grazing points at each tool position are connected with a piecewise linear curve to form the grazing curves at a particular tool position, and then the grazing curves are connected to form a piecewise linear approximation to the swept surface.

The method for computing grazing points on a circle of the tool is illustrated for a truncated cone on the left in Figure 1. For each circular slice of the cone, we find the point $Q$ on the tool axis such that each segment from a point on the circle to $Q$ is perpendicular to the tool surface. Next, we take the plane perpendicular to the direction of motion $\vec{d}$ of $Q$, and intersect this plane with our circle. The intersection points are grazing points.

The basic idea of why this method works is illustrated in the right half of Figure 1. In this figure, we see that we can place a sphere whose center is $Q$ and is tangent to the cone at all point on our circular slice of the cone. The tool motion is a translation plus rotation, which can be expressed relative to any point in space, and relative to $Q$ and a line through $Q$ in particular. Thus, the motion $\vec{d}_{P}$ of a point $P$ differs from $\vec{d}$ (the motion of $Q$ ) only by a rotation $\vec{R}_{Q}$ about a line through $Q: \vec{d}_{P}=\vec{d}+\vec{R}_{Q}$. Since $\vec{R}_{Q}$ comes from a rotation around $Q, \vec{R}_{Q}$ lies in the tangent plane of the sphere at $P$, which will also lie in the tangent plane of the cone at $P$. By intersecting this circle with a plane perpendicular to $\vec{d}$, we obtain those points $P$ such that $\vec{d}_{P}$ lies in the tangent plane of the cone at $P$, i.e., the grazing points. For more details on this computation, see [6].

\section{Extension to elliptic cylinders}

In this section, we extend the grazing curve technique to elliptic cylinders. In this paper, we will only consider finding grazing points on the elliptic cylinder; for details on how to stitch these points into a volume, see our earlier work $[5,6]$.

We reduce the problem of finding grazing points on the elliptic cylinder to that of finding points on ellipse by slicing the elliptic cylinder with planes perpendicular to the tool axis. The method described in the previous section for finding the grazing point on a circular slice of a surface of revolution works because the normals to the surface along each circle pass through the axis of revolution. This allowed us to express the direction of motion of a point on the surface as the sum of the motion of a point on the axis plus a rotation around that point on the axis.

This simple method for finding grazing points fails for an elliptic cylinder because the normals of a slice of the elliptic cylinder do not pass through center of ellipse. Worse, in the case of a twisted cylinder, the normals to the surface do not lie in the plane of the elliptical slice. However, these problems are readily resolved by setting up a small system of equations that we can solve for the grazing points on an elliptic slice. We begin by solving the problem for the elliptic cylinder, after which we will show how to generalize the solution to the twisted elliptic cylinder. 


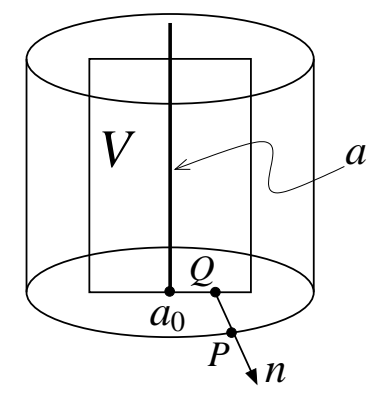

Figure 2: Elliptic Cylinder.

\subsection{Elliptic Cylinder}

If we look at the normal to the elliptic cylinder, it will pass through a point on the major axis of an ellipse on the elliptic cylinder. What we want is a formula for the direction of motion for all points on the major axis plane of the elliptic cylinder. Then, for any position of the elliptic cylinder and any elliptic slice of the elliptic cylinder, we can construct the lines through points $P$ on the ellipse in the direction of the normal. We can intersect these lines with the major axis of the ellipse giving points $Q$. For each $P, Q$ pair, the motion of $P$ differs from $Q$ only by a rotation around $Q$. Thus, to find grazing points we solve for those points on the ellipse where the direction of motion of $Q$ is perpendicular to the normal to the elliptic cylinder at the corresponding $P$.

Our construction is illustrated in Figure 2. We construct the elliptic cylinder by sweeping the ellipse along an axis $a$ that we parameterize with $u$. The equation for the elliptic cylinder (whose axis initially aligns with the $z$-axis) is

$$
C_{0}(u, \theta)=\left[\begin{array}{lll}
A \cos (\theta) & B \sin (\theta) & u
\end{array}\right]^{t},
$$

where $2 A$ is the diameter of the major axis and $2 B$ is the diameter of the minor axis.

The $a$ axis of our cylinder actually has two parameters: $u$, a linear parameter along the axis, and $v$, which describes the motion of the axis through space. For 5-axis machining, knowing the motion of the axis was sufficient to compute grazing points for surfaces of revolution. However, for our method for elliptic cylinders we need to know the motion of the plane containing the major axis of the ellipses of the elliptic cylinder along the entire path of elliptic cylinder.

Let $a_{0}(v)$ be the path followed by the center of the bottom of the elliptic cylinder. Let $V(u, w)$ be a plane with $u$ aligned with $a$ (the axis of the elliptic cylinder) and $w$ aligned with the major axis of the ellipse. Our interest is in the motion of $V$ as the elliptic cylinder moves along its path.

To find this motion, we construct a moving coordinate frame at $a_{0}(v)=a(0, v)$. We align $a$ with the $z$-axis of our local frame; a second axis is given by the cross product of $a$ and $a_{0}^{\prime}(v)$; and the third axis is perpendicular to the first two:

$$
\begin{aligned}
f_{z} & =\hat{a} \\
f_{x} & =\hat{a} \times a_{0}^{\prime} /\left|a \times a_{0}^{\prime}\right| \\
f_{y} & =\hat{a} \times f_{x},
\end{aligned}
$$

where $\hat{a}$ is the unit vector aligned with $a$. We now find the location of $V$ as the elliptic cylinder is swept along $a_{0}(v)$ (we call this moving plane the focal plane $F$ ):

$$
F(u, v, w)=a_{0}+f_{x} V_{x}+f_{y} V_{y}+f_{z} V_{z} .
$$

The velocity of the focal plane is given by the derivative of this equation with respect to $v$. Similarly, we find the equation for the swept cylinder:

$$
C(u, \theta, v)=a_{0}+f_{x} C_{0 x}+f_{y} C_{0 y}+f_{z} C_{0 z} .
$$

The normal to the elliptic cylinder is given by

$$
n=\frac{\partial C}{\partial \theta} \times \frac{\partial C}{\partial u} .
$$

Since our equations involve solving for the zeros of the dot product of $n$ with $V$, there is no need to make $n$ unit length. 


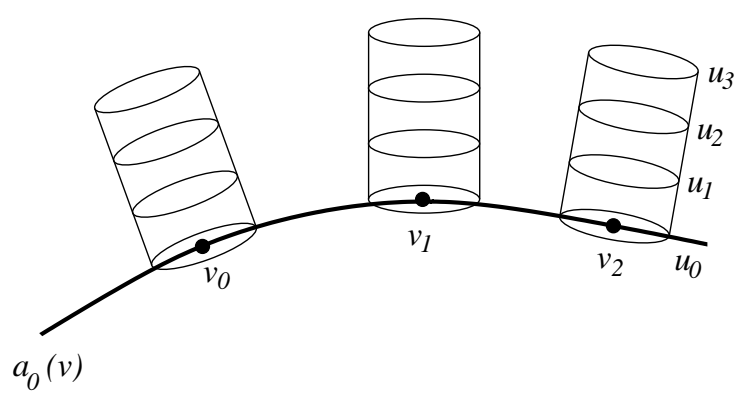

Figure 3: Iterate over $v, u$.

Let $L$ be the line along the surface normal at point $P$ on the elliptic cylinder: $L=P+n t$ where $t$ is a parameter. Intersect the line $L$ with the $V$ plane. That is, $V=L$ will give us three equations and can be used to solve for $u, v$ and $\theta$.

To compute the grazing points on a grazing curve of the surface, set up two for loops, one for $v$ and the other for $u$ and solve $V \cdot n=0$ for $\theta$ as shown in Figure 3. The $v$ parameter is set once for each position of the elliptic cylinder along its path. The $u$ parameter iterates through the slices of the elliptic cylinder. For each $v$, we obtain a set of points that lie upon the surface of the elliptic cylinder at its position for that particular value of $v$. In general, these points will lie on two curves on the elliptic cylinder, and must be joined to form a piecewise linear approximation to each grazing curve, and then the grazing curves must be joined to form a piecewise linear approximation to the swept surface.

While the equations may appear difficult to solve, note that for fixed $u$ and $v$ they simplify greatly. In particular, for fixed $u, v$, the equation for $a_{0}$ is evaluated to a point and a vector (the latter for the derivative of $a_{0}$ ); the frame vectors $f_{x}, f_{y}, f_{z}$ are constant, $F$ (Equation 1 ) and the derivative of $F$ are only functions of $w$; and the equation for the slice of the elliptic cylinder (Equation 2) and the normal to this slice (Equation 3) are functions only of $\theta$. Thus, complexities of the motion of the elliptic cylinder through space (i.e., the $a(u, v)$ function) are simplified away; we are left solving only the equation of an ellipse, the normal to the ellipse, and a linear equation describing the motion of $V$.

\section{$3.2 \quad$ Twisted Elliptic Cylinder}

The mathematics for the twisted elliptic cylinder can be derived merely by applying a twist function to $C$ (Equation 2). We define a twist function $R$ as

$$
R=\left[\begin{array}{ccc}
\cos (\beta(u)) & -\sin (\beta(u)) & 0 \\
\sin (\beta(u)) & \cos (\beta(u)) & 0 \\
0 & 0 & 1
\end{array}\right]
$$

The function $\beta$ gives the twist; if $\beta$ is constant, then we obtain an elliptic cylinder. If we make $\beta$ a function of $v$, then we can rotate the twisted cylinder relative to the direction of motion as the twisted cylinder follows the trajectory of $a_{0}(v)$.

The twisted, elliptic cylinder is given by $R C$. Likewise, we twist $V$ as $V R$. To intersect our lines $L$ with $V$, we may need to extend the definition of $V$ beyond the top/bottom of the elliptic cylinder.

The normal is given by

$$
n=\frac{\partial(R C)}{\partial \theta} \times \frac{\partial(R C)}{\partial u}
$$

In the case of the twisted cylinder, this will be a "twisted plane" (a twisted ribbon).

While most of the mathematics of the previous section carries through without problem, see Section 5 for a discussion of problems that occur in concave regions. Note also that we need to give a new parameter for the axis direction of $V$, since when we use $u$ to select a slice of the twisted cylinder will need values of $F(u, v, w)$ at a range $u$ values.

\section{Examples}

To test our method, we implemented it in the symbolic algebra package Maple [7]. The trajectory of an object exhibiting 5-axis motion is complex as it is a function of the geometry of the machine. To illustrate this work, the 

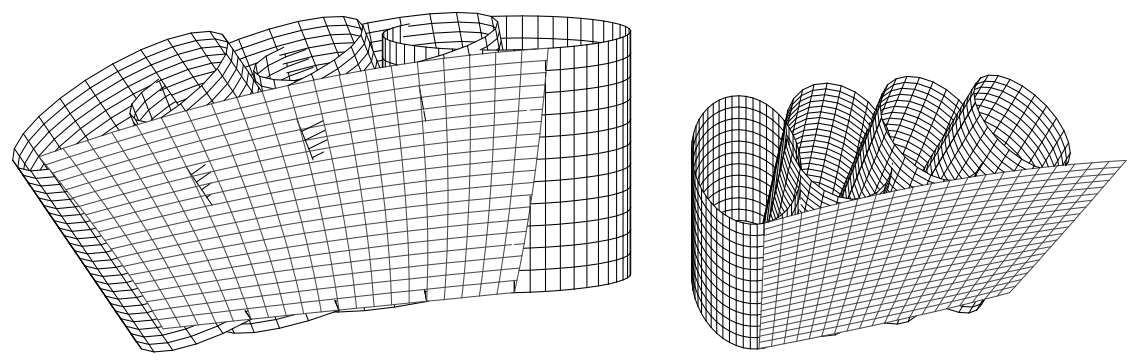

Figure 4: Surfaces swept by elliptic cylinders.
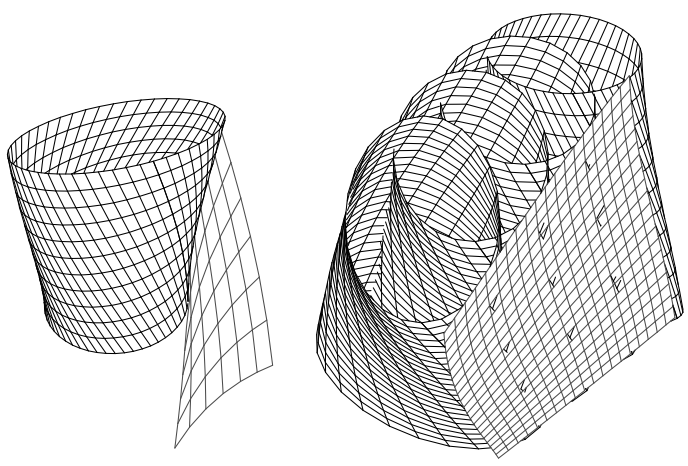

Figure 5: Surfaces swept by twisted elliptic cylinders.

trajectory of $a$ is assumed to be a linear interpolation of the two angles and the linear axis. In Figure 4, we see two surfaces swept by elliptic cylinders. We have placed a few instances of the sweeping surface to indicate its motion.

Although there are further complications if we use the method directly on a twisted elliptic cylinder, testing shows that our new method produces reasonable results as illustrated in Figure 5. However, in these examples, the problems with convex regions discussed in the next section did not occur.

\section{Discussion}

The method described in the previous section only computes the portion of the surface swept by the sides of a truncated elliptic cylinder. To close the surface, we must sweep the top and bottom ellipses. The two curves along the sides of the elliptic cylinder split the top ellipse into two elliptic arcs. For any location of the elliptic cylinder, only one of these arcs is part of the swept surface. Details on selecting which arc to connect, and how to stitch together the piecewise linear approximations to the swept curves can be found in our earlier papers, along with details on handling degenerate cases $[5,6]$.

Although we implemented and test our method on elliptic cylinders, it is clear that the method works for any surface that can be constructed as a sequence of ellipses. For example, we could use our method to find grazing curves on ellipsoids. Generalizations to surfaces having other cross sections should also be possible, but it should be noted that complications occur in regions that are not convex.

In particular, in locally concave regions, a grazing point as defined above may or may not be a point on the swept surface. If the point is in a concave region of positive Gaussian curvature, then it will never be a point on the swept surface. If a grazing point in a region of negative Gaussian curvature, we need to examine the direction on motion to determine if it is on the swept surface. If this vector differentially enters the surface, then the grazing point is a point on the swept surface; otherwise, it is not.

\section{References}

[1] D. Blackmore, M.C. Leu, and L.P. Wang. The sweep-envelope differential equation algorithm and its application to NC machining verification. Computer Aided Design, 29(9):629-637, 1997. 
[2] W.P. Wang and K.K. Wang. Geometric modeling for swept volume of moving solids. IEEE Computer Graphics and Applications, 6(12):8-17, 1986.

[3] Karim Abdel-Malek and Harn-Jou Yeh. Geometric representation of the swept volume using jacobian rankdeficiency conditions. Computer Aided Design, 29(6):457-468, 1997.

[4] Yun C. Chung, Jung W. Park, Hayong Shin, and Byoung K. Choi. Modeling the surface swept by a generalized cutter for NC verification. Computer Aided Design, 30(8):587-594, 1998.

[5] D. Roth, S. Bedi, F. Ismail, and S. Mann. Surface swept by a toroidal cutter during 5-axis machining. Computer Aided Design, 33(1):57-63, 2001.

[6] S. Mann and S. Bedi. Generalization of the imprint method to general surfaces of revolution for NC machining. To appear in Computer Aided Design.

[7] M.B. Monagan, K.O. Geddes, G. Labahn, and S. Vorketter. The Maple Programming Guide. Springer-Verlag, 1996. 